\title{
Replicated evidence for aminoacylase 3 and nephrin gene variations to predict antihypertensive drug responses
}

\begin{abstract}
Aim To replicate the genome-wide associations of the antihypertensive effects of bisoprolol and losartan in GENRES, using the Finnish patients of LIFE study. Patients \& methods: We analyzed association of four SNPs with atenolol and three SNPs with losartan response in 927 Finnish LIFE patients ( 467 for atenolol and 460 for losartan). Results: rs2514036, a variation at a transcription start site of $A C Y 3$, was associated with blood pressure response to atenolol in men in LIFE. Response to bisoprolol was correlated to baseline plasma levels of $\mathrm{N}$-acetylphenylalanine and phenylalanine (ACY3 substrate and end product, respectively) in GENRES study. NPHS1 variation rs3814995 was associated with losartan effect in LIFE. Conclusion: We provide support for two pharmacogenomic markers for beta-blockers and angiotensin receptor antagonists.
\end{abstract}

First draft submitted: 20 December 2016; Accepted for publication: 19 January; Published online: 29 March 2017

Keywords: aminoacylase $3 \bullet$ atenolol $\bullet$ bisoprolol $\bullet$ genome-wide $\bullet$ hypertension $\bullet$ losartan - nephrin • pharmacogenomics

In the past few decades, hypertension has become the leading risk factor for global disease burden through its contribution to cardiovascular morbidity and mortality, as well as total mortality [1]. By effective drug treatment of hypertension it is possible to decrease the amount of cardiovascular events to the extent closely paralleling the blood pressure (BP) response achieved [2]. On average, the main groups of antihypertensive agents are equally effective on lowering BP, but there is a wide variation in individual antihypertensive drug responsiveness [3]. The different antihypertensive drugs are not equal in their efficacy in preventing specific cardiovascular outcomes [2], and many international guidelines for hypertension therapy recommend medication to be chosen based on coexisting conditions [4]. It is frustrating that despite the wide selection of antihypertensive drugs available, only $40-50 \%$ of hypertensive patients seem to achieve adequate BP control while on drug therapy [5].
Genomic differences are proposed to constitute one of the factors explaining the individual variation of $\mathrm{BP}$ responses to drug treatment [6-8]. Searches for genetic variants associating with antihypertensive drug therapies have evolved from candidate gene studies to genome-wide association studies (GWAS), with moderate success [6-11]. Most studies have suffered from methodological or analytical problems, including an insufficient sample size, lack of blinding, use of office BP measurements only and limited use of replication materials. It is possible that population differences may explain some of the failed attempts to replicate certain genomic associations to antihypertensive drug responsiveness [7], which may justify use of patient cohorts coming from genetically more homogeneous populations.

To diminish possible influence of the genetic admixture of the study population [12], we decided to replicate the results of our previous pharmacogenomic three-stage
Jenni M Rimpelä1,2, Kimmo K Kontula ${ }^{* 1,2}$, Frej Fyhrquist ${ }^{3}$, Kati M Donner ${ }^{4}$, Annukka M Tuiskula², Antti-Pekka Sarin ${ }^{4}$, Robert P Mohney ${ }^{5}$, Steven M Stirdivant ${ }^{5}$ \& Timo $P$ Hiltunen ${ }^{1,2}$

'Department of Medicine, University of Helsinki, Finland

${ }^{2}$ Helsinki University Hospital, Helsinki, Finland

${ }^{3}$ Minerva Foundation Institute for Medical Research, Helsinki, Finland ${ }^{4}$ Institute for Molecular Medicine Finland, University of Helsinki, Finland ${ }^{5}$ Metabolon, Inc., Durham, NC, USA *Author for correspondence: Tel.: +358500850260 kimmo.kontula@hus.fi 
GWAS (GENRES study) [10] in Finnish participants to another antihypertensive drug study (LIFE) [13]. Of the four classes of drugs used in GENRES (beta-blocker, diuretic, calcium channel blocker and angiotensin II type 1 receptor antagonist), we were able to replicate data on beta-blocker (bisoprolol in GENRES, atenolol in LIFE) and angiotensin receptor antagonist (losartan in both GENRES and LIFE). As the variants to be replicated in LIFE, we chose the SNPs showing the strongest associations, as also supported by replication data in the Italian and US populations, with BP response to bisoprolol (rs2514036, rs7984003, rs7268800 and rs2765115) and losartan (rs3814995, rs4953045 and rs12814605) in 228 hypertensive Finnish men [10].

\section{Patients \& methods}

Short description of the GENRES study

The GENRES study is the only randomized, doubleblind, cross-over, placebo-controlled pharmacogenomic study in which four classes of antihypertensive drugs (a diuretic, beta-blocker, calcium channel blocker and angiotensin receptor antagonist), each as a monotherapy, were studied in a rotational fashion, using both office and ambulatory BP recordings [10,14]. The study was completed in 228 Finnish men. The performance of the GENRES platform was validated, for example, by pairwise correlations of antihypertensive responses of different antihypertensive drug classes and correlations of baseline plasma renin activities to individual drug responses [14,15]. A recent GWAS of GENRES revealed three SNPs in the same locus associating with a specific drug response with a genome-wide significance $\left(\mathrm{p}<5 \times 10^{-8}\right)$ and 39 SNPs in 30 different loci with a suggestive significance $\left(\mathrm{p}<1 \times 10^{-5}\right)$ [10].

\section{LIFE study}

The LIFE study design and main findings have been described elsewhere [13]. In brief, LIFE was a prospective multicenter (Scandinavia, UK, USA), double-blind, double-dummy, randomized, active-controlled study that was designed to evaluate the long-term effects of losartan compared with atenolol in 9193 hypertensive patients with signs of left ventricle hypertrophy (LVH) in ECG. Patients aged 55-80 with office systolic BP (SBP) of $160-200 \mathrm{mmHg}$ or diastolic BP (DBP) of 95-115 mmHg and signs of LVH in ECG were eligible to participate in the study. After withdrawal of all antihypertensive drugs and 2 weeks on placebo treatment, the study subjects were randomized to receive either losartan- or atenolol-based antihypertensive regimen. Target BP was $140 / 90 \mathrm{mmHg}$ or lower, and the antihypertensive medication was titrated according to the study scheme to achieve the goal. Initially, the treatment consisted of either $50 \mathrm{mg}$ of losartan or $50 \mathrm{mg}$ of atenolol. If the BP target was not reached, $12.5 \mathrm{mg}$ of hydrochlorothiazide was added. If needed, the dosage of the study drug was escalated to $100 \mathrm{mg}$ and last the dosage of hydrochlorothiazide was escalated to $25 \mathrm{mg}$, or other antihypertensive agents were added. The study subjects were followed for a mean of 4.8 years for the primary end points of stroke, myocardial infarction and cardiovascular death. A prespecified pharmacogenetic substudy [16] was conducted among 3503 Scandinavian participants of the LIFE study to test whether candidate gene polymorphisms affected BP or heart rate reduction, cardiovascular events or response to treatment. The 1146 Finnish participants of the LIFE pharmacogenetic sample were available for the present study. The main treatment protocol of the LIFE study and the protocol of the genetics substudy were approved by local ethical committees, and all participants gave a written informed consent. Office BP and drug treatment data were recorded at baseline and at 2, 4 and 6 months after the beginning of the treatment and later on at 6-month intervals. To achieve resemblance to the design of the GENRES study, we focused on BP responses at 2 months in patients who were using monotherapy of $50 \mathrm{mg}$ of atenolol or $50 \mathrm{mg}$ of losartan, or a combination therapy of either of them plus $12.5 \mathrm{mg}$ of hydrochlorothiazide.

\section{Genotyping}

The DNA samples of 1146 LIFE study subjects were genotyped at the Institute for Molecular Medicine Finland, University of Helsinki (FIMM) using the Illumina HumanOmniExpress BeadChip ${ }^{\circledR}$ (Illumina, CA, USA), the same platform as used in the GENRES study [10]. Quality control was performed for the data obtained, and 69 patients were excluded because of low success rates $(<95 \%)$, discrepancy in gender check or demonstration of first-degree relativeness. An additional 116 patients, who at the 2 -month visit were not using monotherapy of $50 \mathrm{mg}$ of the study drug, or a combination therapy of either plus $12.5 \mathrm{mg}$ of hydrochlorothiazide, were excluded from the present analyses. Furthermore, 34 patients, who were either morbidly obese (BMI $>40$ ), had stage four chronic kidney disease (glomerular filtration rate $<30 \mathrm{ml} / \mathrm{min} / 1.73 \mathrm{~m}^{2}$, or urine microalbumin $>300 \mathrm{mg} / \mathrm{l}$ ) or had a BP response residual deviating more than four standard deviations from the mean, were excluded from the analyses. The genotypes of the SNPs used in this study were tested for Hardy-Weinberg equilibrium and no deviation was observed.

\section{Metabolomic measurements}

The metabolomic analysis was performed in EDTA plasma from 35 GENRES patients who had completed 
the whole four-drug study, and who had complete ambulatory 24-h BP response data to bisoprolol, with $>600$ known metabolites measured across diverse and broadly defined biochemical classes including amino acids and peptides (171 biochemicals), carbohydrates (20 biochemicals), energy/tricarboxylic acid cycle intermediates (eight biochemicals), lipids (260 biochemicals), nucleotides (27 biochemicals), vitamins and cofactors (25 biochemicals) and xenobiotics (106 biochemicals). Thus, the results presented here are based on 175 plasma samples, four from placebo and one from bisoprolol periods for each patient.

Nontargeted mass spectrometry (MS) analysis was performed at Metabolon, Inc. (NC, USA). EDTAanticoagulated plasma samples were stored at $-80^{\circ} \mathrm{C}$ until processed. Sample preparation and metabolite extraction were carried out as described previously [17]. The metabolites were identified and quantified using ultra high-performance LC-MS/MS [18,19]. A detailed description of the metabolomic analysis is provided in the Supplementary Methods.

\section{Statistical analysis}

The baseline demographics and BP responses of the study subjects were analyzed using IBM SPSS Statistics 22.0. Fisher's exact test and Student's $t$-test were used to compare the clinical characteristics of the GENRES and the LIFE study subgroups. A statistical approach similar to the discovery GWAS (GENRES study) was used to test the SNP associations in LIFE [10]. SBP and DBP response residuals were generated separately for both atenolol and losartan using linear regression adjusting for age, sex, baseline BP, BMI and use of hydrochlorothiazide at 2 months (Supplementary Table 1). In the second step, current smoking and serum creatinine were tested using stepwise regression and included if significant $(\mathrm{p}<0.10)$. In the LIFE study, covariate-adjusted atenolol BP response residuals were analyzed for associations with the four bisoprolol-associated SNPs derived from the GENRES GWAS, using the linear regression and additive genetic model in PLINK v1.07 [20], and likewise covariate-adjusted losartan residuals were analyzed for associations with the three losartan-associated SNPs derived from the GENRES study. The rationale for the selection of SNPs for the current study is described in Supplementary Table 2. Because the study subjects of the discovery GWAS were all men, a separate association analysis was performed in men if $\mathrm{SNP} \times$ sex interaction was significant $(\mathrm{p}<0.05)$. As there was previous evidence for the associations, successful replication was defined as $\mathrm{p}<0.05$ with effect in the same direction as in the initial study. A more conservative approach, using Bonferroni correction, would have required a nominal two-sided p-value of $0.014(=0.05 / 7 \times 2)$, based on seven SNPs and requirement of same direction of effect and taking into account the fact that systolic and diastolic responses are highly correlated.

Power calculations for the genetic replication analyses of the seven SNPs in LIFE were carried out using program Quanto, v. 1.2.4 [21]. The power estimates for reaching two-sided $p$-values of $<0.05$ and $\beta$-values obtained in GENRES ranged from 56 to $99 \%$ (Supplementary Table 3).

In the metabolomic analyses of the GENRES patients, normalized values of metabolite concentrations were used. The mean level of four placebo periods was used as the baseline level (a similar approach was used for the BP data in GENRES). For Pearson correlation analyses of metabolite baseline levels with placebo BP levels and bisoprolol BP responses, covariateadjusted residuals were generated; the tested variables in stepwise regression were age, BMI, current smoking, serum creatinine, fasting serum glucose and daily urinary sodium excretion after the first placebo period of the study. Using $\mathrm{p}<0.10$ as a criterion, serum creatinine was selected as a covariate for plasma phenylalanine concentration (standardized $\beta=0.37 ; \mathrm{p}=0.02$ ), daily urinary sodium excretion for $\mathrm{N}$-acetylphenylalanine (standardized $\beta=-0.36 ; \mathrm{p}=0.03$ ) and fasting serum glucose for $\mathrm{N}$-acetyltyrosine (standardized $\beta=0.35 ; \mathrm{p}=0.03$ ). None of the tested variables was significant for plasma tyrosine concentration. In the correlation analyses, residuals were also used for placebo BP levels and bisoprolol BP responses. They were generated, correspondingly, using the whole GENRES population. Age and serum creatinine were used as covariates for placebo ambulatory SBP level, but none was significant for placebo DBP level. Placebo baseline SBP level and current smoking were used as covariates for bisoprolol SBP response and placebo DBP level for bisoprolol DBP response, as described previously [10]. Bisoprolol-induced metabolite concentration changes were analyzed with paired $t$-test.

\section{Results}

Study subjects

A detailed description of the study subjects of the GENRES study is given in our previous publications $[10,14,15]$. The main characteristics are also listed in Table 1.

Clinical characteristics of the LIFE study subgroups are described in Table 1. A total of 927 Finnish men and women were included in the genetic analysis of the present study; 467 in the atenolol group and 460 in the losartan group. The baseline characteristics were similar in both treatment arms. Approximately half of the patients were men and the mean age was 64 years. For 
Table 1. Clinical characteristics of study participants.

\begin{tabular}{|c|c|c|c|c|}
\hline \multirow[t]{2}{*}{ Parameter } & \multicolumn{2}{|c|}{ LIFE participants } & \multicolumn{2}{|c|}{ GENRES participants } \\
\hline & Atenolol & Losartan & Bisoprolol & Losartan \\
\hline $\mathrm{n}$ & 467 & 460 & 207 & 202 \\
\hline Age (years) & $64 \pm 6.2$ & $64 \pm 6.2$ & $51 \pm 6.2^{*}$ & $51 \pm 6.3 *$ \\
\hline Men $(\%)$ & 47 & 49 & $100 *$ & $100 *$ \\
\hline \multicolumn{5}{|l|}{ At baseline } \\
\hline BMI $\left(\mathrm{kg} / \mathrm{m}^{2}\right)$ & $27.6 \pm 3.6$ & $27.9 \pm 3.6$ & $26.7 \pm 2.7 *$ & $26.6 \pm 2.8^{*}$ \\
\hline Office SBP $(\mathrm{mmHg})$ & $171 \pm 14$ & $171 \pm 14$ & $152 \pm 13^{*}$ & $152 \pm 13^{*}$ \\
\hline Office DBP (mmHg) & $99 \pm 7$ & $98 \pm 8$ & $100 \pm 7$ & $100 \pm 7 *$ \\
\hline Ambulatory $24-\mathrm{h}$ SBP $(\mathrm{mmHg})$ & NA & NA & $135 \pm 10$ & $135 \pm 10$ \\
\hline Ambulatory $24-\mathrm{h}$ DBP $(\mathrm{mmHg})$ & NA & NA & $93 \pm 6$ & $93 \pm 5$ \\
\hline Current smoker (\%) & 10 & 10 & $15^{*}$ & $16^{*}$ \\
\hline Serum creatinine $(\mu \mathrm{mol} / \mathrm{l})$ & $81 \pm 13$ & $81 \pm 14$ & $86 \pm 13^{*}$ & $86 \pm 13^{*}$ \\
\hline \multicolumn{5}{|c|}{ At 2 months (LIFE)/4 weeks (GENRES) } \\
\hline$\triangle$ Office SBP $(\mathrm{mmHg})$ & $-16.4 \pm 14.8$ & $-16.5 \pm 13.9$ & $-12.9 \pm 10.7 *$ & $-9.2 \pm 12.0 *$ \\
\hline$\triangle$ Office DBP $(\mathrm{mmHg})$ & $-10.7 \pm 10.0$ & $-8.4 \pm 7.9$ & $-9.7 \pm 7.2$ & $-7.3 \pm 6.9$ \\
\hline$\triangle$ Ambulatory 24 -h SBP $(\mathrm{mmHg})$ & NA & NA & $-11.1 \pm 6.2$ & $-9.1 \pm 6.7$ \\
\hline$\triangle$ Ambulatory $24-\mathrm{h}$ DBP $(\mathrm{mmHg})$ & NA & NA & $-8.3 \pm 4.2$ & $-6.1 \pm 4.7$ \\
\hline On hydrochlorothiazide (\%) & 57 & 57 & $0 *$ & $0 *$ \\
\hline Duration of treatment (days) & $61(47-79)$ & $61(45-78)$ & $30(27-56)^{*}$ & $30(27-46) *$ \\
\hline
\end{tabular}

the purpose of the present study, timing of the antihypertensive response was set at 2 months; in both groups the median length of the study drug therapy was 61 days (range: 47-79 days in the atenolol group and 45-78 days in the losartan group) and 57\% of the patients were using hydrochlorothiazide at the 2-month visit. In the atenolol group, mean baseline BP was $171 / 99 \mathrm{mmHg}$ and the mean BP response was $-16.4 /-10.7 \mathrm{mmHg}$, while in the losartan group the corresponding figures were $171 / 98 \mathrm{mmHg}$ and $-16.5 /-8.4 \mathrm{mmHg}$. The most noticeable differences between the GENRES and the LIFE study subjects at baseline are higher age, higher BP level (which is placebo level in GENRES) and inclusion of women in the LIFE study.

Replication analysis of BP response to losartan Table 2 shows the p-values from the linear regression analysis of the BP response to losartan in the LIFE study. Of the three SNPs (rs3814995, rs4953045 and rs12814605) that showed the strongest associations with losartan response in the GENRES study, rs3814995 on chromosome 19 was associated with DBP response to losartan $(\beta=-0.9 \mathrm{mmHg} ; \mathrm{p}=0.04$; proportion of variation in the BP response residual explained: 0.009) with the same direction of effect in the LIFE study (Figure 1).
For SBP response, the effect was in the same direction, but the association was not statistically significant. In patients using losartan $50 \mathrm{mg}$ as monotherapy, the associations were similar $(\mathrm{n}=199 ; \beta=-1.3 \mathrm{mmHg}$, $\mathrm{p}=0.21$ for SBP response and $\beta=-0.9 \mathrm{mmHg}, \mathrm{p}=0.13$ for DBP response). According to Ensembl database (www.ensembl.org) rs3814995 is located in the exon 3 of the NPHS1 gene in chromosome 19 coding for the glomerular protein nephrin and is predicted to cause an amino acid substitution of glutamic acid to lysine (p.Glu117Lys). The other two SNPs did not show statistically significant replication. SNP-sex interactions were not significant for any of the tested SNPs.

Replication analysis of BP response to atenolol To validate the four SNPs (rs2514036, rs7984003, rs7268800 and rs2765115) that showed association with bisoprolol response in the GENRES study, we tested for their associations with atenolol response in LIFE. Table 3 shows the $p$-values from the linear regression analysis of the BP response to atenolol. rs2514036, achieving genome-wide significance $\left(\mathrm{p}=2 \times 10^{-8}\right)$ in GENRES, associated with systolic $(\beta=-5.5 \mathrm{mmHg}$; $\mathrm{p}=0.005$; proportion of variation in the BP response residual explained: 0.04) and diastolic $(\beta=-1.9 \mathrm{mmHg}$; 
$\mathrm{p}=0.05$; proportion of variation in the $\mathrm{BP}$ response residual explained: 0.02) $\mathrm{BP}$ response to atenolol in men of the LIFE study, with the effect in the same direction as in GENRES (Figure 2). When women were included in the analysis, the association was no longer statistically significant, but the effect was still in the same direction. In patients with atenolol $50 \mathrm{mg}$ as monotherapy at 2 months, a corresponding association was found for SBP response in both men and women $(\mathrm{n}=201 ; \beta=-3.6 \mathrm{mmHg} ; \mathrm{p}=0.04)$ and in men only $(\mathrm{n}=105 ; \beta=-4.0 \mathrm{mmHg} ; \mathrm{p}=0.13)$. The other three SNPs tested did not show a statistically significant replication. According to Ensembl database, rs2514036 (base variation G/A) corresponds exactly to the second transcription initiation site of the $A C Y 3$ gene, coding for $\mathrm{ACY} 3$, which is known to deacetylate mercapturic acids and $\mathrm{N}$-acetyl aromatic amino acids [22].

\section{Metabolic phenotypes related to ACY3 polymorphism \& beta-blocker responses}

The fact that we were able to replicate the significant association of the rs2514036 (present in the ACY3 gene) with bisoprolol responses in GENRES to the atenolol responses in LIFE prompted us to carry out an analysis of the levels and drug responses of certain aromatic amino acids relevant to ACY3 action. We have carried out a comprehensive metabolic profiling using ultra high-performance LC-MS/MS (data to be presented in detail in a separate report) in 175 plasma samples available from 35 patients in GENRES, using bisoprolol as the beta-blocker. In the present study, we restricted our analysis on the compounds $\mathrm{N}$-acetylphenylalanine and $\mathrm{N}$-acetyltyrosine, and their deacetylated products (derived by ACY3 enzyme action) phenylalanine and tyrosine, respectively [22].

We found a significant inverse correlation between the ambulatory DBP (but not SBP) level on placebo, and baseline concentrations of plasma phenylalanine $(r=-0.56 ; \mathrm{p}=0.0005)$ and tyrosine $(\mathrm{r}=-0.47$; $\mathrm{p}=0.003$ ) (Figure 3). In addition, the SBP and DBP responses to bisoprolol were significantly correlated to the plasma baseline (placebo) levels of phenylalanine ( $\mathrm{p}=0.03$ and 0.0005 , respectively) and $\mathrm{N}$-acetylphenylalanine ( $\mathrm{p}=0.0009$ and 0.01 , respectively) (Figure 4); the correlations were in the same direction but weaker for plasma tyrosine ( $p=0.06$ and 0.05 , respectively) and $\mathrm{N}$-acetyltyrosine ( $\mathrm{p}=0.22$ and 0.38 , respectively).

In addition, the plasma concentrations of these compounds increased slightly upon bisoprolol administration (Supplementary Figure 2); there was no relation between these changes and antihypertensive responses to bisoprolol (data not shown).

Recently, Yu et al. [23] reported a genome-wide significant association of rs12288023, another SNP of the

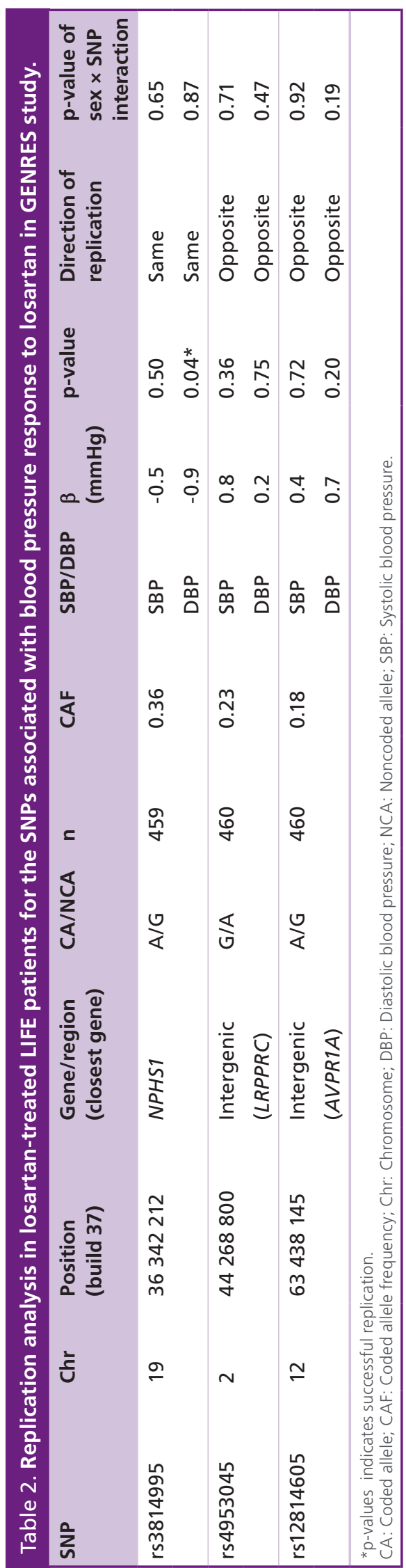



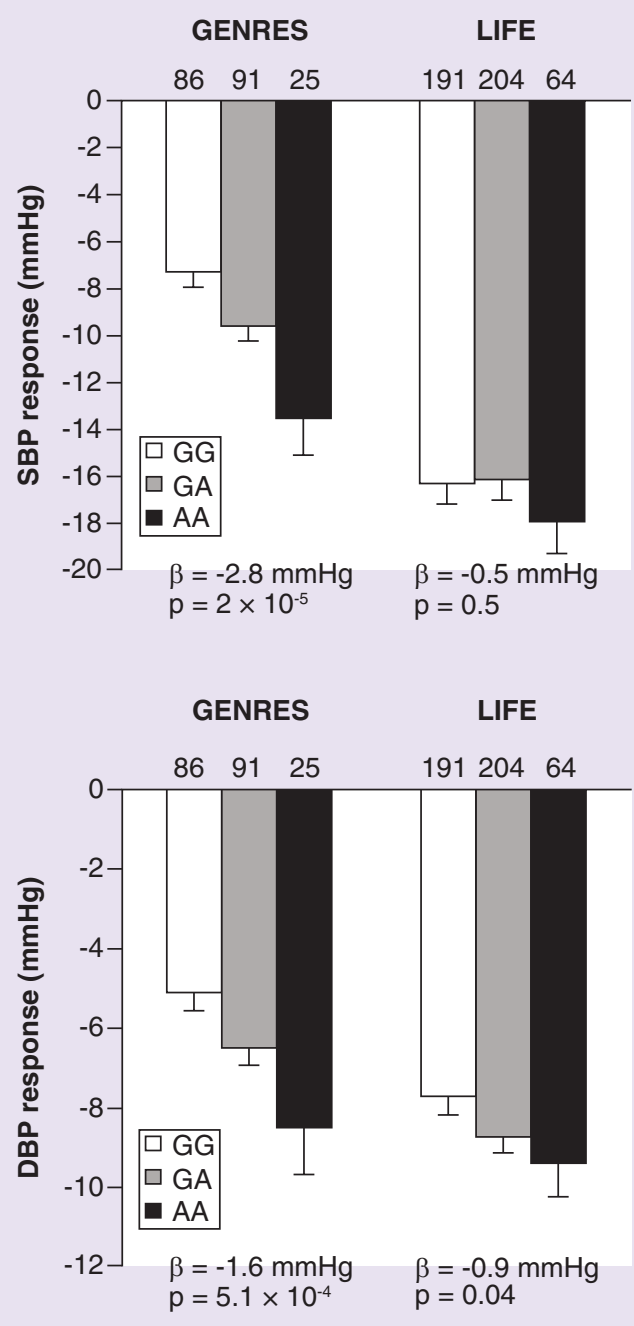

Figure 1. Blood pressure response to losartan by genotype for rs3814995 in NPHS1 on chromosome 19 in the discovery cohort (GENRES) and replication study (LIFE). The number of subjects in each genotype is shown at the top. Blood pressure responses are adjusted for significant covariates as described in the 'Patients \& methods' section. Error bars indicate standard error of means.

DBP: Diastolic blood pressure; SBP: Systolic blood pressure.

$A C Y 3$ gene, with serum $\mathrm{N}$-acetylphenylalanine levels ( $\mathrm{p}=9 \times 10^{-15} ; \mathrm{n}=15,700$ subjects). This SNP is in linkage disequilibrium with $\mathrm{rs} 2514036\left(\mathrm{r}^{2}=0.50\right)$, as studied in Finns of the 1000 Genomes Project [24]. We studied rs12288023 by imputation in GENRES (imputation quality, $\left.\mathrm{r}^{2}=0.97\right)$ and found that it was associated with SBP and DBP responses to bisoprolol $\left(\mathrm{p}=3.1 \times 10^{-5}\right.$ and $3.4 \times 10^{-5}$, respectively) and modestly with placebo plasma $\mathrm{N}$-acetylphenylalanine levels $(\mathrm{p}=0.24)$, similar to our top SNP in GENRES, rs2514036 ( $\mathrm{p}=0.17$ ) (Supplementary Figure 3). The association of the different rs2514036 genotypes with both $\mathrm{N}$-acetylphenyl- alanine placebo level and SBP response to bisoprolol is illustrated in Supplementary Figure 4.

\section{Discussion}

The present combined pharmacogenomic study provides additional evidence for two gene loci influencing responsiveness to antihypertensive drugs, one locus for beta-blockers and another for angiotensin receptor antagonists. We used the GENRES study as our primary data set because it has been designed for the particular purpose of pharmacogenomic analysis. The nature of GENRES as a randomized, double-blind and monotherapy-rotational study makes it an almost ideal platform to generate hypotheses, with the inevitable limitation of its sample size. We had only moderate success in our attempts to replicate GENRES data in materials from USA and Italy [10], which prompted us to pick up a replication sample from our own population. In fact, due to a variety of reasons, including its geographical position, historical population bottlenecks and linguistic borders, Finns are considered to represent a population markedly distinct from other western populations in their genetic terms [25]. Although this is in particular true for the presence or absence of rare gene mutations, it may also influence studies on common genetic variants.

The present replication study carried out in the LIFE material strengthens our previous evidence of the association of rs3814995 with losartan responsiveness in hypertensive patients. Accordingly, hypertensive patients carrying the A allele (corresponding to lysine) of rs3814995 are more likely to have favorable response to losartan compared with those carrying the $\mathrm{G}$ allele (corresponding to glutamic acid). In the initial GWAS, the association of rs3814995 with losartan response showed replication in two populations; the GERA II study ( $p=0.03 / 0.02$ for SBP/DBP responses) and the SOPHIA study ( $p=0.19 / 0.03$ for SBP/DBP response) [10]. This makes LIFE study the fourth independent population to show a similar association. In contrast, the two other SNPs (rs4953045 and rs12814605) did not show any significant association with losartan responses in LIFE.

rs3814995 is a missense variant in the coding region of NPHS1 gene on chromosome 19q13.12, causing the substitution of glutamic acid to lysine (p.Glu117Lys) in nephrin, the principal structural protein of the glomerular podocytes [26]. Structure databases PolyPhen2 [27] and SIFT [28] predict this alteration to be probably damaging. Linkage analysis of GENRES GWAS data (Supplementary Figure 5) points to NPHS1 as the causal gene. As cited before [10], specific mutations of NPHS1 are known to cause the congenital nephrotic syndrome of the Finnish type (for review, see [29]). 
There is increasing evidence from laboratory animals and some data from human experimental models to link nephrin, angiotensin and angiotensin receptor antagonists together. Thus, in a hypertensive rat model, angiotensin II was found to decrease nephrin gene expression [30], while irbesartan [31], valsartan [32] and losartan [33] have all been shown to counteract the decrease of nephrin levels in diabetic hypertensive rats. Furthermore, candesartan was found to abolish stretch-induced nephrin downregulation in cultured human glomerular podocytes [34]. There appear to be only few data on the possible clinical significance of the Glu117Lys variation. When studying patients with diabetic proteinuria, Pettersson-Fernholm et al. noticed that carriers of the Lys117 allele had a later onset of diabetes than its noncarriers [35]. Summarizing, there is increasing evidence, derived from the present study and a combination of three other studies (GENRES, GERA II and SOPHIA) [10] that the nephrin Glu117Lys variation serves as a marker of the antihypertensive response of angiotensin II receptor blockers. In the present study, Lys117Lys homozygotes showed $2-6 \mathrm{mmHg}$ stronger $\mathrm{SBP}$ and $2-3 \mathrm{mmHg}$ stronger DBP reductions than the Glu117Glu homozygotes (Figure 1). Any underlying mechanism remains, however, to be investigated.

The other principal finding of the present study was the replication of the association of rs 2514036 variation with beta-blocker response in Finns; this association was initially discovered in GENRES comprising men only and was subsequently confirmed in LIFE, and here principally in men. Accordingly, heterozygous AG men showed approximately $5 \mathrm{mmHg}$ greater reduction in SBP and 2-3 mmHg greater reduction in DBP compared with the AA homozygotes in response to bisoprolol or atenolol administration; the single GG homozygote men in GENRES showed even greater responses (Figure 2); there were no GG homozygote men in LIFE. It is of note that this association of rs 2514036 with beta-blocker responses could not be replicated in the North American population sample of the PEAR study [10]. However, sex-dependent metabolic effects of atenolol responsiveness were also revealed in the PEAR study: in atenolol-treated subjects, development of impaired fasting glucose was associated with baseline plasma phenylalanine levels in men [36].

According to Ensembl database rs2514036 is a 5'-variant of the ACY3 gene in chromosome 11q13.2. In GENRES GWAS data, the SNPs with the lowest p-values are located in $A C Y 3$ and are also in high linkage disequilibrium with the top SNP rs2514036 (Supplementary Figure 6). According to Ensembl database, $A C Y 3$ encodes two different transcript variants, ACY3-001 consisting of 8 exons and ACY3-002 con-

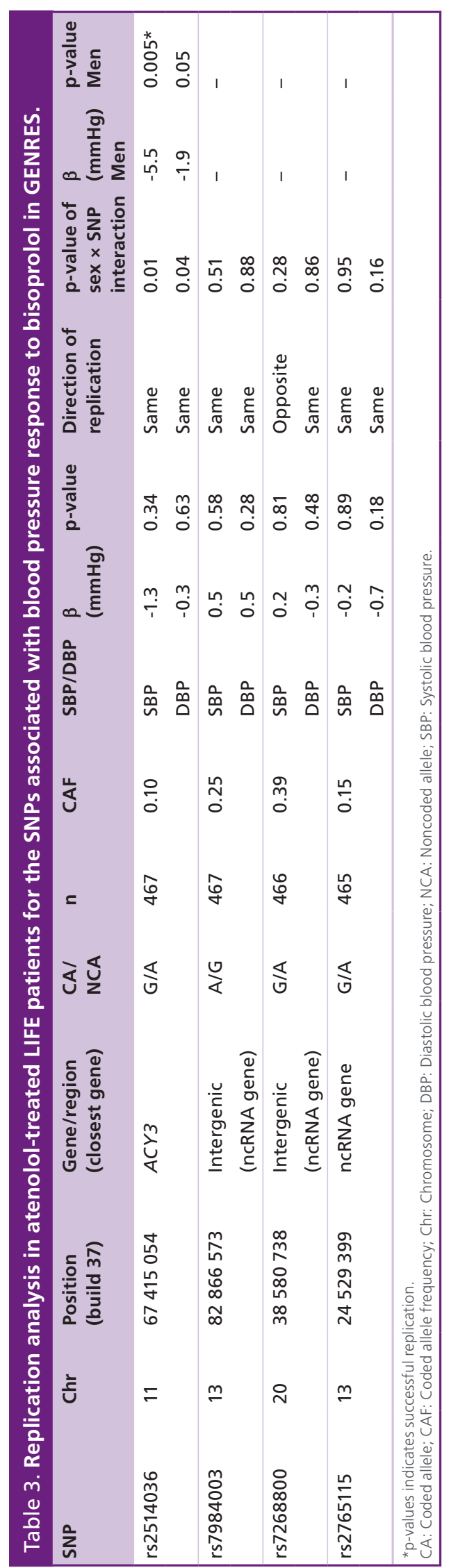



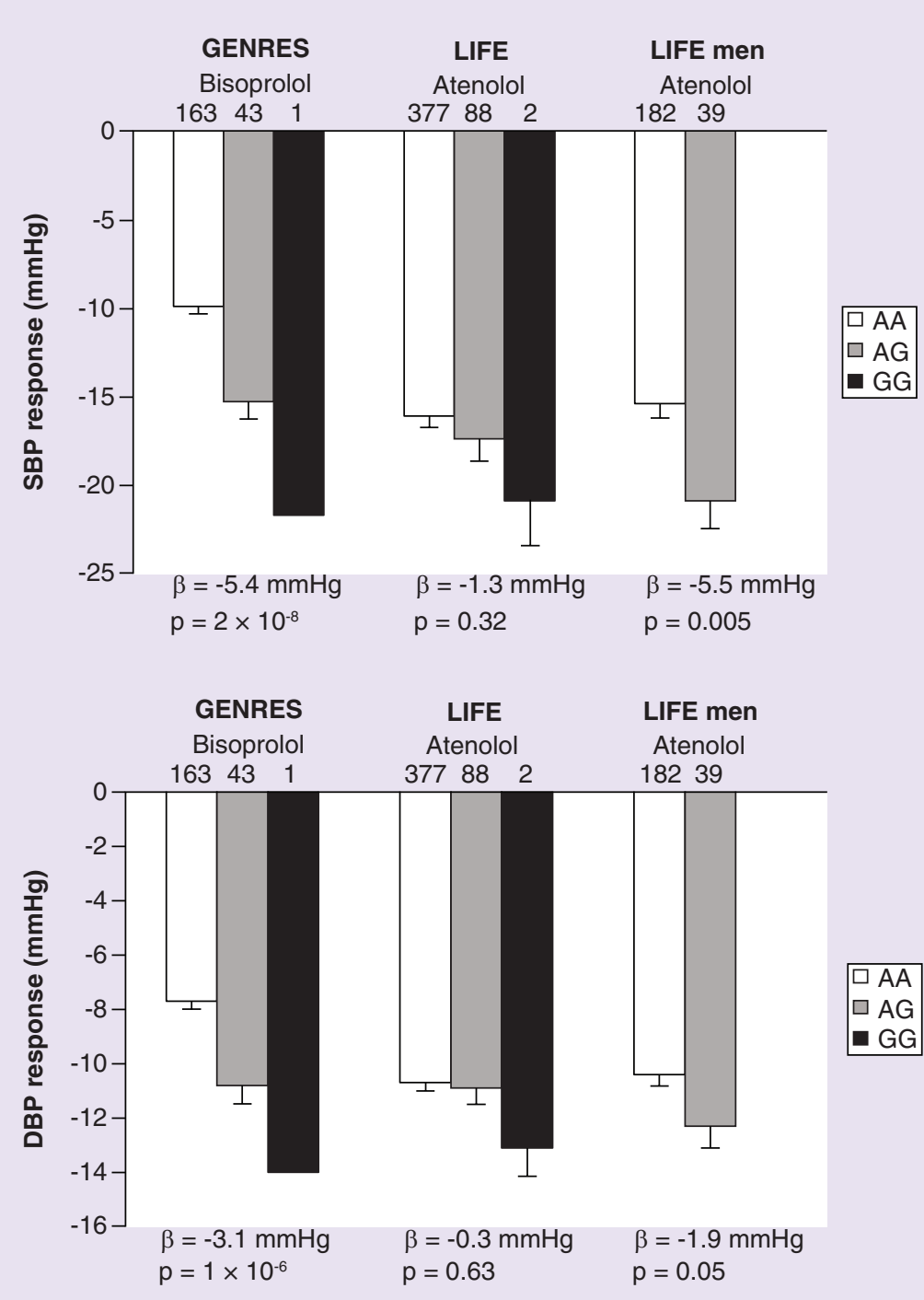

Figure 2. Blood pressure response to bisoprolol in the discovery cohort (GENRES) and atenolol in the replication study (LIFE) by genotype for rs 2514036 in ACY3 on chromosome 11. The number of subjects in each genotype is shown at the top. Blood pressure responses are adjusted for significant covariates as described in the 'Patients \& methods' section. Error bars indicate standard error of means. DBP: Diastolic blood pressure; SBP: Systolic blood pressure.

sisting of 7 exons. The protein product of transcript 001 contains 319 amino acids while the protein from transcript 002 is shorter, consisting of 198 amino acids. Interestingly, rs 2514036 hits exactly the transcription initiation site of ACY-002. The nucleotide change from the wild-type A-G changes a Forkhead box (Fox) transcription factor family DNA-binding domain sequence (reviewed in [37]) from ATAAATA to ATGAATA, thus possibly affecting $A C Y 3$ expression.

A search of publicly available gene expression data sets for SNPs associated with ACY3 expression showed further evidence for this locus. First, in the in the Genotype-Tissue Expression database [38], rs2514036 was strongly associated with ACY3 expres- sion $\left(\mathrm{p}=1.8 \times 10^{-20}\right.$ in meta-analysis of all available 37 tissues, each with more than 70 analyzed samples) [39]. Second, rs $431359323 \mathrm{~kb}$ upstream from rs2514036 $\left(r^{2}=0.50 ; D^{\prime}=1.00\right.$ in Finnish 1000 Genomes population) was associated with $A C Y 3$ expression $\left(\mathrm{p}=1.3 \times 10^{-7}\right)$ in lymphoblastoid cell lines of the MRCE data set $(n=550)[40,41]$. This SNP was imputed in GENRES and was associated with both systolic $\left(\beta=-5.4 \mathrm{mmHg} ; \mathrm{p}=3.1 \times 10^{-5}\right)$ and diastolic $\left(\beta=-3.6 \mathrm{mmHg} ; \mathrm{p}=5.1 \times 10^{-5}\right)$ bisoprolol responses. Third, rs 2514036 was associated with ACY3 expression $\left(\mathrm{p}=3.5 \times 10^{-3}\right.$, combined result of ten regions of the brain) in the Braineac database [42,43], and, last, in 173 ileal biopsy specimen $\left(\mathrm{p}=9.3 \times 10^{-8}\right)[44]$. 
$A C Y 3$ encodes aminoacylase 3 , an enzyme deacetylating mercapturic acids and $\mathrm{N}$-acetylated aromatic amino acids and, as studied in mouse tissues, its highest expression is found in the kidney, followed by liver, heart and brain [22]. In humans, $A C Y 3$ expression has been shown in neurons and adrenal medulla [45]. Mercapturic acids are condensation products formed by coupling of cysteine with aromatic compounds and are considered to represent useful markers of exposure to exogenous industrial chemicals; they do not appear to show any obvious connection to human hypertension and its drug treatment. In contrast, there is alluring evidence linking $A C Y 3$ and aromatic amino acid metabo- lism to regulation of blood pressure. First, as cited above, the tissue-specific expression pattern of $A C Y 3$ is compatible with its potential role in blood pressure regulation. Second, its ability to deacetylate N-acetylphenylalanine to phenylalanine and $\mathrm{N}$-acetyltyrosine to tyrosine [46] could link this enzyme to the synthesis chain of catecholamines. Third, in the present study we report a significant correlation between the SBP and DBP responses to bisoprolol and pretreatment (placebo) plasma levels of phenylalanine and $\mathrm{N}$-acetylphenylalanine (Figure 4). Last, serum phenylalanine level as such has been associated with increased cardiovascular risk: a prospective study in three population-

\section{A Placebo SBP vs plasma phenylalanine and tyrosine}
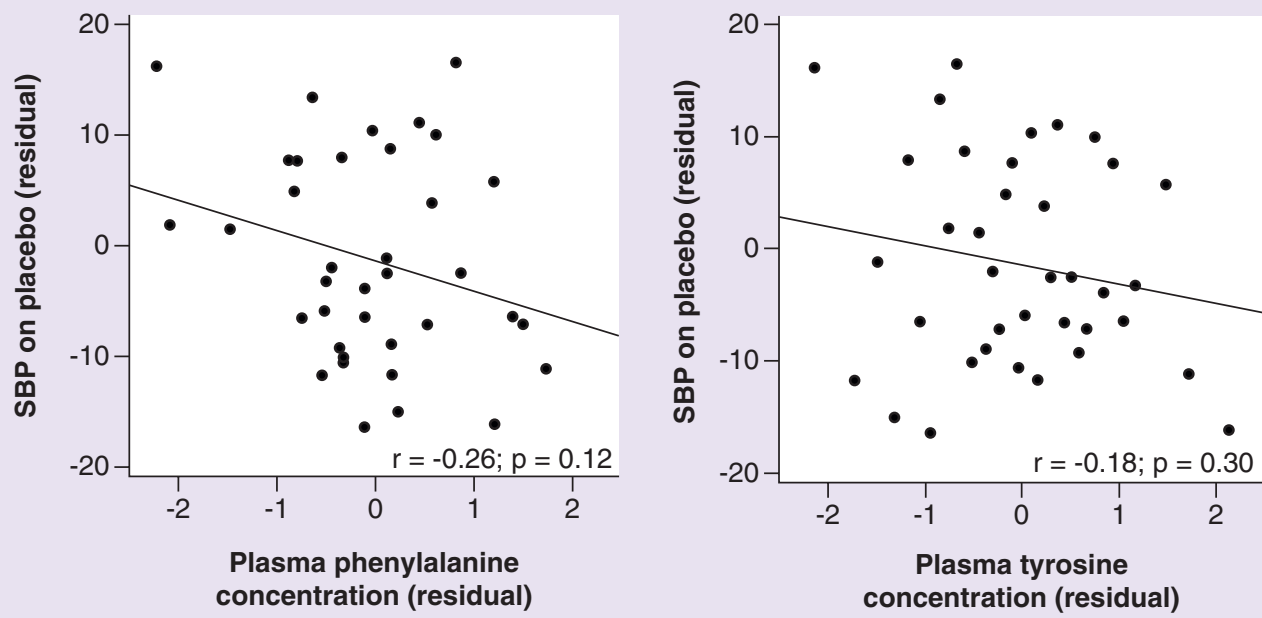

(B)

Placebo DBP vs plasma phenylalanine and tyrosine
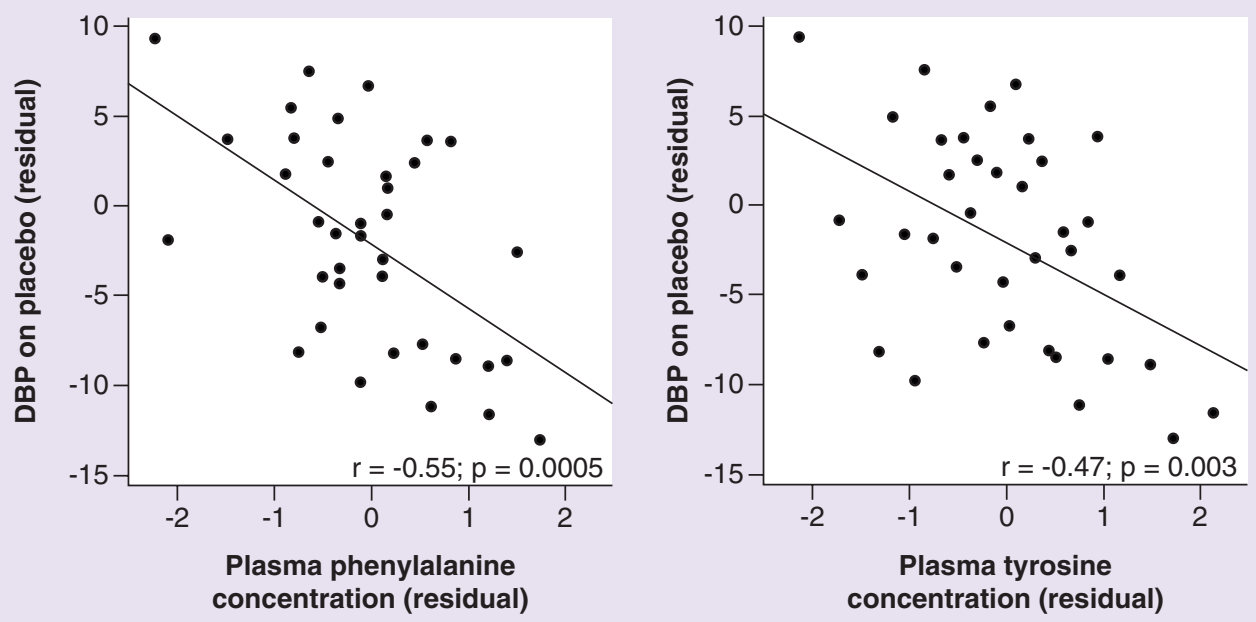

Figure 3. Association of plasma phenylalanine and tyrosine levels with ambulatory blood pressure level in GENRES subjects on placebo. (A) SBP; (B) DBP. Blood pressure levels and normalized metabolite levels are presented as covariate-adjusted residuals that were calculated as described in the 'Patients \& methods' section. DBP: Diastolic blood pressure; SBP: Systolic blood pressure. 


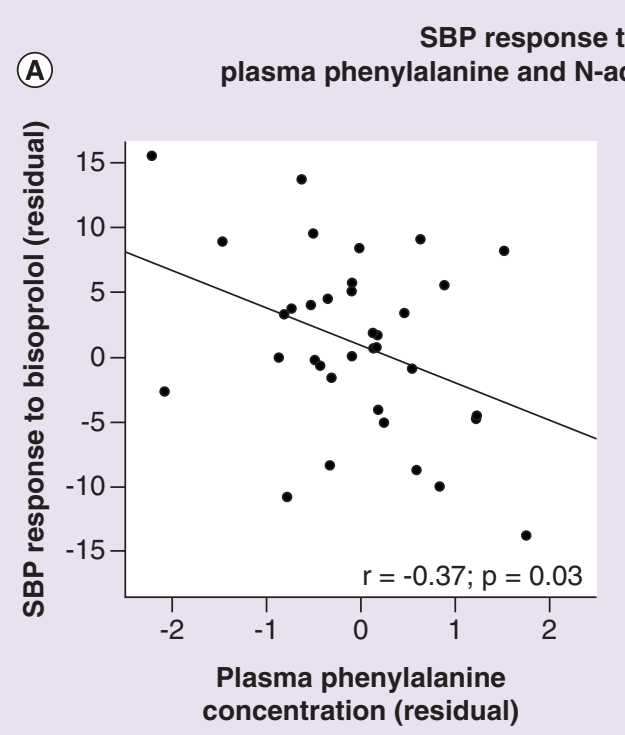

SBP response to bisoprolol vs

(A) plasma phenylalanine and $\mathrm{N}$-acetylphenylalanine on placebo

DBP response to bisoprolol vs
(B) plasma phenylalanine and $\mathrm{N}$-acetylphenylalanine on placebo
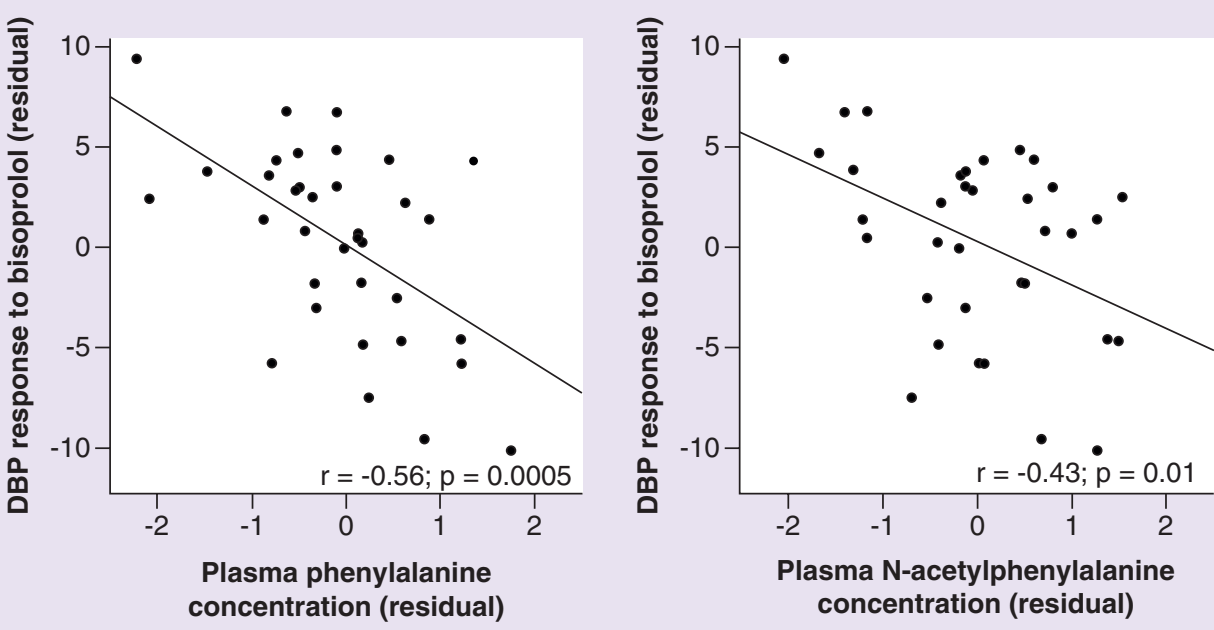

Figure 4. Association of placebo plasma phenylalanine and $\mathrm{N}$-acetylphenylalanine levels with ambulatory blood pressure response to bisoprolol in GENRES patients. (A) SBP response; (B) DBP response. Blood pressure responses and normalized metabolite levels are presented as covariate-adjusted residuals that were calculated as described in the 'Patients \& methods' section.

DBP: Diastolic blood pressure; SBP: Systolic blood pressure.

based cohorts showed that higher serum phenylalanine level was associated with $18 \%$ increased cardiovascular risk upon long-term follow-up [47], and elevated serum phenylalanine concentrations have also been linked to increased risk of Type 2 diabetes mellitus [48-50], as well as pre-eclampsia [51].

The present study is not without certain important limitations. First, when analyzing GENRES data we have systematically used ambulatory BP measurements, due to their better repeatability (lower coefficient of variation) compared with office BP measurements [14], while we were obliged to use the latter technique in the LIFE study. Second, the GENRES study was conducted in men only whereas both sexes were almost equally represented in LIFE. Third, while losartan was used in both GENRES and LIFE, we compared bisoprolol in GENRES with atenolol in LIFE. Fourth, approximately half of the LIFE participants were also using small doses of thiazide diuretics. However, in the monotherapy groups, the observed associations were similar, albeit less significant due to lower numbers of subjects. Fifth, according to inclusion criteria of LIFE, 
these patients had high-risk hypertension with electrocardiographic evidence of LVH, while GENRES patients had uncomplicated hypertension. All these limitations listed above may play a role in lack of replication of some of the positive SNPs (revealed in GENRES) in the LIFE material. Sixth and last, the replication results of rs3814995 in LIFE (losartan arm) met the nominal $\mathrm{p}$-value limit of $<0.05$ but not the Bonferroni-corrected limit of $<0.014$. We consider this, however, a positive replication because the analysis was directed to the previous evidence from three different studies.

\section{Conclusion}

We have carried out a replication study of the most significant genomic associations of beta-blocker and angiotensin II receptor antagonist responses initially revealed in the GENRES study [10], using the corresponding data in the LIFE study. In both studies, hypertensive patients of Finnish origin only were investigated. Our data provide strengthening evidence for the $A C Y 3$ gene variation as a biomarker of betablocker responsiveness and the NPHS1 gene variation as a marker of angiotensin II type 1 receptor antagonist responsiveness. We took the advantage of performing these analyses in a genetically homogeneous population in order to decrease noise possibly caused by major ancestral differences of the patients. It remains to be shown, however, whether the data can be generalized to other populations, even if we had pre-existing information from GENRES suggesting positive replication of nephrin variation and losartan responses in other populations. The genotype-related differences in SBP $(2-7 \mathrm{mmHg})$ and DBP $(2-3 \mathrm{mmHg})$ responses found in the present study should be sufficient to be taken into account in personalized attempts to decrease the risk of cardiovascular mortality of hypertensive patients [10,52].

\section{Supplementary data}

To view the supplementary data that accompany this paper, please visit the journal website at: www.futuremedicine.com/ doi/pdf/10.2217/pgs-2016-0204

\section{Acknowledgements \\ The authors thank S Saarinen for excellent technical help.}

\section{Financial \& competing interests disclosure}

The present study was supported by grants from 'The Sigrid Juselius Foundation' and 'The Finnish Foundation for Cardiovascular Research'. AP Sarin is currently an employee at Blueprint Genetics, Inc., and, as such, has affiliations with or financial involvement with Blueprint Genetics, Inc. RP Mohney and SM Stirdivant are employees of Metabolon, Inc. and, as such, have affiliations with or financial involvement with Metabolon, Inc. The authors have no other relevant affiliations or financial

\section{Executive summary}

\section{Aim}

- This study was undertaken to replicate and extend the positive genome-wide associations of the antihypertensive effects of a beta-blocker (bisoprolol) and an angiotensin receptor antagonist (losartan) in the GENRES study, using the Finnish arm of the LIFE study.

Replication analysis of blood pressure response to losartan

- NPHS1 (encoding the kidney protein nephrin) variation rs3814995 (Glu117Lys) was associated with the antihypertensive effect of losartan in the LIFE study (diastolic blood pressure [BP] change, $p=0.04$ ), supporting the initial findings in the GENRES, GERA II and SOPHIA studies.

\section{Replication analysis of $\mathrm{BP}$ response to atenolol}

- $A C Y 3$ (encoding for aminoacylase 3 ) variation $r 52514036$ was associated with systolic $(p=0.005)$ and diastolic $(p=0.05)$ BP responses to atenolol in men of the LIFE study, supporting the genome-wide significant association $\left(p=2 \times 10^{-8}\right)$ obtained from the GENRES study, which included only men.

- eQTL data in four different databases show strong association of rs 2514036 with ACY3 expression in several tissues.

Metabolic phenotypes related to $A C Y 3$ polymorphism \& beta-blocker responses

- The plasma concentrations of the substrate ( $\mathrm{N}$-acetylphenylalanine) and the end product (acetylphenylalanine, a precursor of catecholamines) of ACY3 action were analyzed in GENRES.

- Systolic and diastolic BP responses to bisoprolol were significantly correlated to the plasma baseline (placebo) levels of phenylalanine ( $p=0.03$ and 0.0005 , respectively) and $N$-acetylphenylalanine $(p=0.0009$ and 0.01 , respectively) in GENRES.

\section{Conclusion}

- The results strengthen the evidence for the $A C Y 3$ variation rs2514036 as a biomarker of beta-blocker responsiveness and the NPHS1 variation rs3814995 as a marker of angiotensin II type 1 receptor antagonist responsiveness.

- The plasma concentrations of $\mathrm{N}$-acetylphenylalanine and phenylalanine, ACY3-related catecholamine synthesis precursors, may predict the antihypertensive response to beta-blockers. 
involvement with any organization or entity with a financial interest in or financial conflict with the subject matter or materials discussed in the manuscript apart from those disclosed.

No writing assistance was utilized in the production of this manuscript.

\section{Ethical conduct of research}

The authors state that they have obtained appropriate institutional review board approval and have followed the principles

\section{References}

Papers of special note have been highlighted as:

- of interest; $\bullet \bullet$ of considerable interest

1 Lim SS, Vos T, Flaxman AD et al. A comparative risk assessment of burden of disease and injury attributable to 67 risk factors and risk factor clusters in 21 regions, 1990-2010: a systematic analysis for the Global Burden of Disease Study 2010. Lancet 380 (9859), 2224-2260 (2012).

2 Ettehad D, Emdin CA, Kiran A et al. Blood pressure lowering for prevention of cardiovascular disease and death: a systematic review and meta-analysis. Lancet 387(10022), 957-967 (2016).

3 Dickerson JE, Hingorani AD, Ashby MJ, Palmer CR, Brown MJ. Optimisation of antihypertensive treatment by crossover rotation of four major classes. Lancet 353(9169), 2008-2013 (1999).

- A rotational study on optimization of antihypertensive treatment.

4 ESH/ESC Task Force for the Management of Arterial Hypertension. 2013 Practice guidelines for the management of arterial hypertension of the European Society of Hypertension (ESH) and the European Society of Cardiology (ESC): ESH/ESC task force for the management of arterial hypertension. J. Hypertens. 31(10), 1925-1938 (2013).

5 Bromfield SG, Bowling CB, Tanner RM et al. Trends in hypertension prevalence, awareness, treatment, and control among US adults 80 years and older, 1988-2010. J. Clin. Hypertens. (Greenwich) 16(4), 270-276 (2014).

6 Arwood MJ, Cavallari LH, Duarte JD. Pharmacogenomics of hypertension and heart disease. Curr. Hypertens. Rep. 17(9), 586 (2015).

7 Cooper-DeHoff RM, Johnson JA. Hypertension pharmacogenomics: in search of personalized treatment approaches. Nat. Rev. Nephrol. 12(2), 110-122 (2016).

8 Manunta P, Ferrandi M, Cusi D, Ferrari P, Staessen J, Bianchi G. Personalized therapy of hypertension: the past and the future. Curr. Hypertens. Rep. 18(3), 24 (2016).

9 Kamide K, Asayama K, Katsuya T et al. Genome-wide response to antihypertensive medication using home blood pressure measurements: a pilot study nested within the HOMED-BP study. Pharmacogenomics 14(14), 1709-1721 (2013).

10 Hiltunen TP, Donner KM, Sarin AP et al. Pharmacogenomics of hypertension: a genome-wide, placebo-controlled cross-over study, using four classes of outlined in the Declaration of Helsinki for all human and animal experimental investigations. In addition, for the investigations involving human subjects, informed consent has been obtained from the participants involved.

\section{Open access}

This work is licensed under the Attribution-NonCommercialNoDerivatives 4.0 Unported License. To view a copy of this license, visit http://creativecommons.org/licenses/by-nc-nd/4.0/

antihypertensive drugs. J. Am. Heart Assoc. 4(1), e001521 (2015).

- The first pharmacogenomic study on hypertension involving four different drug classes and a double-blind rotational design.

11 Salvi E, Wang Z, Rizzi F et al. Genome-wide and gene-based meta-analyses identify novel loci influencing blood pressure response to hydrochlorothiazide. Hypertension 69 (1), 51-59 (2017).

12 Polimanti R, Iorio A, Piacentini S, Manfellotto D, Fuciarelli M. Human pharmacogenomic variation of antihypertensive drugs: from population genetics to personalized medicine. Pharmacogenomics 15(2), 157-167 (2014).

13 Dahlof B, Devereux RB, Kjeldsen SE et al. Cardiovascular morbidity and mortality in the Losartan Intervention For Endpoint reduction in hypertension study (LIFE): a randomised trial against atenolol. Lancet 359(9311), 995-1003 (2002).

14 Hiltunen TP, Suonsyrja T, Hannila-Handelberg T et al. Predictors of antihypertensive drug responses: initial data from a placebo-controlled, randomized, cross-over study with four antihypertensive drugs (The GENRES Study). Am. J. Hypertens. 20(3), 311-318 (2007).

15 Suonsyrja T, Hannila-Handelberg T, Paavonen KJ et al. Laboratory tests as predictors of the antihypertensive effects of amlodipine, bisoprolol, hydrochlorothiazide and losartan in men: results from the randomized, double-blind, crossover GENRES Study. J. Hypertens. 26(6), 250-1256 (2008).

16 Nordestgaard BG, Kontula K, Benn M et al. Effect of ACE insertion/deletion and 12 other polymorphisms on clinical outcomes and response to treatment in the LIFE study. Pharmacogenet. Genomics 20(2), 77-85 (2010).

17 Evans AM, Bridgewater BR, Liu Q et al. High resolution mass spectrometry improves data quantity and quality as compared with unit mass resolution mass spectrometry in high-throughput profiling metabolomics. Metabolomics 4(2), 132 (2014).

18 Evans AM, DeHaven CD, Barrett T, Mitchell M, Milgram E. Integrated, nontargeted ultrahigh performance liquid chromatography/electrospray ionization tandem mass spectrometry platform for the identification and relative quantification of the small-molecule complement of biological systems. Anal. Chem. 81(16), 6656-6667 (2009).

19 Dehaven CD, Evans AM, Dai H, Lawton KA. Organization of GC/MS and LC/MS metabolomics data into chemical libraries. J. Cheminform. 2(1), 9 (2010). 
20 Purcell S, Neale B, Todd-Brown K et al. PLINK: a tool set for whole-genome association and population-based linkage analyses. Am. J. Hum. Genet. 81(3), 559-575 (2007).

21 Gauderman WJ. Sample size requirements for matched casecontrol studies of gene-environment interaction. Stat. Med. 21(1), 35-50 (2002).

22 Pushkin A, Carpenito G, Abuladze N et al. Structural characterization, tissue distribution, and functional expression of murine aminoacylase III. Am. J. Physiol. Cell Physiol. 286(4), C848-C856 (2004).

23 Yu B, Zheng Y, Alexander D, Morrison AC, Coresh J, Boerwinkle E. Genetic determinants influencing human serum metabolome among African Americans. PLoS Genet. 10(3), e1004212 (2014).

- A genome-wide association study on human metabolome, revealing several interesting associations.

241000 Genomes Project Consortium, Auton A, Brooks LD et al. A global reference for human genetic variation. Nature 526(7571), 68-74 (2015).

25 Kere J. Human population genetics: lessons from Finland. Annu. Rev. Genomics Hum. Genet. 2, 103-128 (2001).

26 Ristola M, Lehtonen S. Functions of the podocyte proteins nephrin and Neph3 and the transcriptional regulation of their genes. Clin. Sci. (Lond.) 126(5), 315-328 (2014).

Adzhubei IA, Schmidt S, Peshkin L et al. A method and server for predicting damaging missense mutations. Nat. Methods 7(4), 248-249 (2010).

28 Kumar P, Henikoff S, Ng PC. Predicting the effects of coding non-synonymous variants on protein function using the SIFT algorithm. Nat. Protoc. 4(7), 1073-1081 (2009). Holmberg C, Jalanko H. Congenital nephrotic syndrome and recurrence of proteinuria after renal transplantation. Pediatr. Nephrol. 29(12), 2309-2317 (2014).

30 Jia J, Ding G, Zhu J et al. Angiotensin II infusion induces nephrin expression changes and podocyte apoptosis. Am. J. Nephrol. 28(3), 500-507 (2008).

31 Bonnet F, Cooper ME, Kawachi H, Allen TJ, Boner G, Cao Z. Irbesartan normalises the deficiency in glomerular nephrin expression in a model of diabetes and hypertension. Diabetologia 44(7), 874-877 (2001).

32 Davis BJ, Cao Z, de Gasparo M, Kawachi H, Cooper ME, Allen TJ. Disparate effects of angiotensin II antagonists and calcium channel blockers on albuminuria in experimental diabetes and hypertension: potential role of nephrin. J. Hypertens. 21(1), 209-216 (2003).

33 Castoldi G, di Gioia CR, Bombardi C et al. Prevention of diabetic nephropathy by compound 21 , selective agonist of angiotensin type 2 receptors, in Zucker diabetic fatty rats. Am. J. Physiol. Renal Physiol. 307(10), F1123-F1131 (2014).

34 Miceli I, Burt D, Tarabra E, Camussi G, Perin PC, Gruden G. Stretch reduces nephrin expression via an angiotensin IIAT(1)-dependent mechanism in human podocytes: effect of rosiglitazone. Am. J. Physiol. Renal Physiol. 298(2), F381-F390 (2010).

35 Pettersson-Fernholm K, Forsblom C, Perola M, Groop PH, FinnDiane Study Group. Polymorphisms in the nephrin gene and diabetic nephropathy in Type 1 diabetic patients. Kidney Int. 63(4), 1205-1210 (2003).

Dehoff RM, Hou W, Weng L et al. Is diabetes mellitus-linked amino acid signature associated with betablocker-induced impaired fasting glucose? Circ. Cardiovasc. Genet. 7(2), 199-205 (2014).

37 Georges AB, Benayoun BA, Caburet S, Veitia RA. Generic binding sites, generic DNA-binding domains: where does specific promoter recognition come from? FASEB J. 24(2), 346-356 (2010).

38 GTExPortal. www.gtexportal.org/home

39 GTEx Consortium. Human genomics. The Genotype-Tissue Expression (GTEx) pilot analysis: multitissue gene regulation in humans. Science 348(6235), 648-660 (2015).

-. Describes data and a website that facilitate analysis of noncoding genetic variations.

40 Liang L, Morar N, Dixon AL et al. A cross-platform analysis of 14,177 expression quantitative trait loci derived from lymphoblastoid cell lines. Genome Res. 23(4), 716-726 (2013).

41 Ma B, Huang J, Liang L. RTeQTL: real-time online engine for expression quantitative trait loci analyses. 2014, bau066 (2014)

42 BRAINEAC. www.braineac.org

43 Ramasamy A, Trabzuni D, Guelfi S et al. Genetic variability in the regulation of gene expression in ten regions of the human brain. Nat. Neurosci. 17(10), 1418-1428 (2014).

44 Kabakchiev B, Silverberg MS. Expression quantitative trait loci analysis identifies associations between genotype and gene expression in human intestine. Gastroenterology 144(7), 1488-1496 (2013).

45 Long PM, Stradecki HM, Minturn JE, Wesley UV, Jaworski DM. Differential aminoacylase expression in neuroblastoma. Int. J. Cancer 129(6), 1322-1330 (2011).

46 Newman D, Abuladze N, Scholz K et al. Specificity of aminoacylase III-mediated deacetylation of mercapturic acids. Drug Metab. Dispos. 35 (1), 43-50 (2007).

47 Wurtz P, Havulinna AS, Soininen P et al. Metabolite profiling and cardiovascular event risk: a prospective study of 3 population-based cohorts. Circulation 131(9), 774-785 (2015).

- Reports the association of serum phenylalanine level with cardiovascular risk.

48 Wang TJ, Larson MG, Vasan RS et al. Metabolite profiles and the risk of developing diabetes. Nat. Med. 17(4), 448-453 (2011).

49 Stancakova A, Civelek M, Saleem NK et al. Hyperglycemia and a common variant of GCKR are associated with the levels of eight amino acids in 9,369 Finnish men. Diabetes 61(7), 1895-1902 (2012).

50 Floegel A, Stefan N, Yu Z et al. Identification of serum metabolites associated with risk of Type 2 diabetes using a targeted metabolomic approach. Diabetes 62 (2), 639-648 (2013). 
51 Odibo AO, Goetzinger KR, Odibo L et al. First-trimester prediction of preeclampsia using metabolomic biomarkers: a discovery phase study. Prenat. Diagn. 31(10), 990-994 (2011).
52 Lewington S, Clarke R, Qizilbash N, Peto R, Collins R, Prospective Studies Collaboration. Age-specific relevance of usual blood pressure to vascular mortality: a meta-analysis of individual data for one million adults in 61 prospective studies. Lancet 360 (9349), 1903-1913 (2002). 